KODAI MATH. SEM. REP.

21 (1969), 463-471

\title{
INTEGRAL FORMULAS FOR SUBMANIFOLDS OF CODIMENSION 2 AND THEIR APPLICATIONS
}

\author{
By Kentaro Yano and Masafumi Okumura
}

\section{§ 1. Introduction}

Various integral formulas for hypersurfaces of a Riemannian manifold have been found and applied to the study of closed hypersurfaces with constant mean curvature.

Integral formulas for submanifolds of codimension greater than 1 was first obtained by Okumura [6] for the case of submanifolds of codimension 2 of an odd dimensional sphere. He made use of the natural contact structure of the odd dimensional sphere. Integral formulas for general submanifolds of a Riemannian manifold have been obtained by Katsurada [1], [2], [3], Kôjyô [2], Nagai [3], [4], and Yano [9].

In a recent paper [7], Okumura obtained integral formulas for a submanifold of codimension 2, invariant under the curvature transformation, of a Riemannian manifold admitting an infinitesimal conformal transformation and used them to prove that, under certain conditions, the submanifold in question is totally umbilical.

In the present paper, we study a problem similar to that treated in [7]. In [7], the ambient Riemannian manifold was supposed to admit an infinitesimal conformal transformation, but in this paper, we assume instead that there exists a vector field along the submanifold whose covariant differential is proportional to the displacement. We do not assume that the submanifold is invariant under the curvature transformation but instead we put a condition on the integral of a quantity depending on the curvature.

We moreover study the case in which the ambient Riemannian manifold admits a scalar function $v$ such that $\nabla_{j} \nabla_{i} v=f(v) g_{j i}$ and prove that the submanifold satisfying certain conditions is isometric to a sphere by a method used in [8].

\section{$\S 2$. Submanifolds of codimension 2 .}

We consider an $(n+2)$-dimensional orientable Riemannian manifold $M^{n+2}$ of differentiability class $C^{\infty}$ covered by a system of coordinate neighborhoods $\left\{U ; x^{h}\right\}$, where and in the sequel the indices $h, i, j, \cdots$ run over the range $\{1,2, \cdots, n, n+1, n+2\}$. We denote by $g_{j i},\left\{{ }_{j}{ }_{i} i\right\}, \nabla_{\imath}$, and $K_{k j i}{ }^{h}$, the metric tensor, the Christoffel symbols formed

Received June 26, 1969. 
with $g_{j i}$, the operator of covariant differentiation with respect to $\left\{{ }_{j}{ }_{i}\right\}$, and the curvature tensor of $M^{n+2}$ respectively.

We consider an $n$-dimensional orientable submanifold $M^{n}$ differentiably imbedded in $M^{n+2}$ and denote by

$$
x^{h}=x^{h}\left(u^{a}\right)
$$

its parametric representation, where and in the sequel the indices $a, b, c, d, e$ run over the range $\{1,2, \cdots, n\}$. If we put

$$
B_{b}{ }^{h}=\partial_{b} x^{h}, \quad\left(\partial_{b}=\partial / \partial u^{b}\right)
$$

then $B_{b}{ }^{h}$, for each fixed $b$, is a vector field tangent to $M^{n}$ and $B_{b}{ }^{h}$ are linearly independent. A Riemannian metric

$$
g_{c b}=g_{j i} B_{c}{ }^{j} B_{b}{ }^{2}
$$

is induced on $M^{n}$. We denote by $\left\{c_{b}^{a}\right\}, \nabla_{c}$ and $K_{d c b}{ }^{a}$, the Christoffel symbols formed with $g_{c b}$, the operator of covariant differentiation with respect to $\left\{\begin{array}{c}a_{b} \\ b\end{array}\right\}$ and the curvature tensor of $M^{n}$ respectively.

Now, the so-called van der Waerden-Bortolotti covariant derivative of $B_{b}{ }^{h}$ is given by

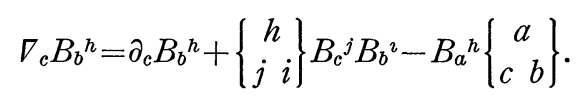

Since $\nabla_{c} B_{b}{ }^{h}$, as vectors of $M^{n+2}$, are normal to $M^{n}$, the vector field

$$
H^{h}=\frac{1}{n} g^{c b} \nabla_{c} B_{b}{ }^{h}
$$

is normal to the submanifold $M^{n}$ and is called the mean curvature vector of $M^{n}$.

We assume throughout the paper that the mean curvature vector never vanishes and take the first unit normal $C^{n}$ to $M^{n}$ in the direction of the mean curvature vector. We take the second unit normal $D^{h}$ in such a way that $B_{1}{ }^{h}, B_{2}{ }^{h}, \cdots, B_{n}{ }^{h}, C^{h}$ and $D^{h}$ give the positive orientation of $M^{n+2}$.

Then the equations of Gauss and those of Weingarten are written as

$$
\nabla_{c} B_{b}{ }^{h}=h_{c b} C^{h}+k_{c b} D^{h}
$$

and

$$
\left\{\begin{array}{l}
\nabla_{c} C^{h}=-h_{c}^{a} B_{a}{ }^{h}+l_{c} D^{h}, \\
\nabla_{c} D^{h}=-k_{c}{ }^{a} B_{a}{ }^{h}-l_{c} C^{h}
\end{array}\right.
$$

respectively, where $h_{c b}$ and $k_{c b}$ are the second fundamental tensors with respect to the normals $C^{h}$ and $D^{h}$ respectively and $l_{c}$ the third fundamental tensor, $h_{c}{ }^{a}$ and $k_{c}{ }^{a}$ being defined by 


$$
h_{c}^{a}=h_{c b} g^{b a}, \quad k_{c}{ }^{a}=k_{c b} g^{b a} .
$$

The normals $C^{h}$ and $D^{h}$ being chosen intrinsically, the quantities $h, k$ and $l$ are all intrinsic quantities of $M^{n}$.

Since $(1 / n) g^{c b} \nabla_{c} B_{b}{ }^{h}$ is in the direction of $C^{h}$, we see from (2.6) that

$$
g^{c b} k_{c b}=k_{c}^{c}=0 .
$$

Now the equations of Gauss, those of Codazzi and those of Ricci are respectively written as

$$
\begin{gathered}
K_{k j i n} B_{d}{ }^{k} B_{c}{ }^{j} B_{b}{ }^{2} B_{a}{ }^{h}=K_{d c b a}-h_{d a} h_{c b}+h_{c a} h_{d b}-k_{d a} k_{c b}+k_{c a} k_{d b}, \\
\left\{\begin{array}{l}
K_{k j i h} B_{d}{ }^{k} B_{c}^{j} B_{b}{ }^{2} C^{h}=\nabla_{d} h_{c b}-\nabla_{c} h_{d b}-l_{d} k_{c b}+l_{c} k_{d b}, \\
K_{k j i h} B_{d}{ }^{k} B_{c}{ }^{j} B_{b}{ }^{i} D^{h}=\nabla_{d} k_{c b}-\nabla_{c} k_{d b}+l_{d} h_{c b}-l_{c} h_{d b},
\end{array}\right. \\
K_{k j i h} B_{d}{ }^{k} B_{c}{ }^{\jmath} C^{i} D^{h}=\nabla_{d} l_{c}-\nabla_{c} l_{d}+h_{d a} k_{c}{ }^{a}-h_{c a} k_{d}{ }^{a} .
\end{gathered}
$$

\section{$\S 3$. Vector fields along the submanifold of codimension 2 .}

Take a normal vector field

$$
V^{h}=\lambda C^{h}+\mu D^{h}
$$

Then, using equations of Weingarten, we have

$$
\nabla_{c} V^{h}=\left(-\lambda h_{c}{ }^{a}-\mu k_{c}{ }^{a}\right) B_{a}{ }^{h}+\left(\partial_{c} \lambda-l_{c} \mu\right) C^{h}+\left(\partial_{c} \mu+l_{c} \lambda\right) D^{h},
$$

and consequently the connection induced in the normal bundle from the Riemannian connection of $M^{n+2}$ is given by

$$
\nabla_{c}^{\prime} \lambda=\partial_{c} \lambda-l_{c} \mu, \quad \nabla_{c}^{\prime} \mu=\partial_{c} \mu+l_{c} \lambda
$$

Thus in order that a normal vector field $\lambda C^{h}+\mu D^{h}$ be parallel with respect to the connection induced in the normal bundle, it is necessary and sufficient that

$$
\partial_{c} \lambda-l_{c} \mu=0, \quad \partial_{c} \mu+l_{c} \lambda=0 .
$$

These equations show that

$$
\lambda^{2}+\mu^{2}=\text { constant, }
$$

that is, a normal vector field parallel with respect to the connection induced in the normal bundle is of constant length.

If $\lambda C^{h}(\neq 0)$ is parallel with respect to the connection induced in the normal bundle, then we have

$$
\lambda=\text { const. and } l_{c}=0 \text {, }
$$


and conversely. If $\mu D^{h}(\neq 0)$ is parallel, we have

$$
\mu=\text { const. and } l_{c}=0 \text {, }
$$

and conversely. Thus, in order that the mean curvature vector $\left.(1 / n) g^{c b} \nabla_{c} B_{b}{ }^{h} \neq 0\right)$ be parallel with respect to the connection induced in the normal bundle, it is necessary and sufficient that

$$
h_{a}{ }^{a}=\text { const. } \neq 0, \quad l_{c}=0 .
$$

Take next a vector field $X^{n}$ defined along the submanifold $M^{n}$ and assume that the covariant differential of this vector field is always proportional to the displacement along the manifold. For such a vector field we have

$$
\nabla_{b} X^{h}=f B_{b}{ }^{h}
$$

$f$ being a scalar function of $M^{n}$.

If we put

$$
X^{h}=z^{a} B^{h}+\alpha C^{h}+\beta D^{h},
$$

we have

$$
\begin{aligned}
\nabla_{b} X^{h}= & \left(\nabla_{b} z^{a}-\alpha h_{b}{ }^{a}-\beta k_{b}^{a}\right) B_{a}^{h} \\
& +\left(\partial_{b} \alpha-l_{b} \beta+h_{b a} z^{a}\right) C^{h} \\
& +\left(\partial_{b} \beta+l_{b} \alpha+k_{b a} z^{a}\right) D^{h} .
\end{aligned}
$$

Thus if we assume that the covariant differential of $X^{h}$ is proportional to the displacement along $M^{n}$, then we have

$$
\nabla_{b} z^{a}=f \delta_{b}^{a}+\alpha h_{b}{ }^{a}+\beta k_{b}{ }^{a}
$$

or

$$
\nabla_{b} z_{a}=f g_{b a}+\alpha h_{b a}+\beta k_{b a}
$$

and

$$
\left\{\begin{array}{l}
\partial_{b} \alpha-l_{b} \beta+h_{b a} z^{a}=0, \\
\partial_{b} \beta+l_{b} \alpha+k_{b a} z^{a}=0 .
\end{array}\right.
$$

\section{§4. Integral formulas for a closed submanifold of codimension 2.}

We consider an $(n+2)$-dimensional Riemannian manifold $M^{n+2}$ and a closed orientable submanifold $M^{n}$ of codimension 2 imbedded in it. We assume that there exists a vector field 


$$
X^{h}=z^{a} B_{a}{ }^{h}+\alpha C^{h}+\beta D^{h}
$$

along $M^{n}$ whose covariant differential along $M^{n}$ is always proportional to the displacement:

$$
\nabla_{c} X^{h}=f B_{c}{ }^{h}
$$

Then we have

$$
\nabla_{c} z_{b}=f g_{c b}+\alpha h_{c b}+\beta k_{c b}
$$

from which

$$
g^{c b} \nabla_{c} z_{b}=n f+\alpha h_{a}^{a} .
$$

Thus, integrating over $M^{n}$, we find

$$
\int_{M^{n}}\left(n f+\alpha h_{a}^{a}\right) d V=0
$$

where $d V$ denotes the volume element of $M^{n}$.

We now compute $\nabla_{a}\left(h_{b}{ }^{a} z^{b}\right)$ :

$$
\begin{aligned}
\nabla_{a}\left(h_{b}{ }^{a} z^{b}\right) & =\left(\nabla_{a} h_{b}{ }^{a}\right) z^{b}+h^{b a} \nabla_{b} z_{a} \\
& =\left(\nabla_{a} h_{b}{ }^{a}\right) z^{b}+h^{b a}\left(f g_{b a}+\alpha h_{b a}+\beta k_{b a}\right) \\
& =\left(\nabla_{a} h_{b}{ }^{a}\right) z^{b}+f h_{a}{ }^{a}+\alpha h^{b a} h_{b a}+\beta h^{b a} k_{b a} .
\end{aligned}
$$

But, from the first of equations (2.10) of Codazzi, we have

$$
K_{k j i h} B_{d}{ }^{k} B^{j i} C^{h}=\nabla_{d} h_{a}{ }^{a}-\nabla_{a} h_{d}{ }^{a}+l_{a} k_{d}{ }^{a},
$$

where

$$
B^{j i}=g^{c b} B_{c}^{j} B_{b}{ }^{2}
$$

and consequently we have

$$
\begin{aligned}
\nabla_{a}\left(h_{b}{ }^{a} z^{b}\right)= & -K_{k j i h} B_{d}{ }^{k} z^{d} B^{j i} C^{h}+z^{d} \nabla_{d} h_{a}{ }^{a}+l_{a} k_{d}{ }^{a} z^{d} \\
& +f h_{a}{ }^{a}+\alpha h^{b a} h_{b a}+\beta h^{b a} k_{b a} .
\end{aligned}
$$

Thus, integrating over $M^{n}$, we obtain

$$
\int_{M^{n}} K_{k j i h} B_{d}^{k} z^{d} B^{j i} C^{h} d V
$$

$$
=\int_{M^{n}}\left(z^{d} \nabla_{d} h_{a}{ }^{a}+l_{a} k_{d}{ }^{a} z^{d}+f h_{a}^{a}+\alpha h^{b a} h_{b a}+\beta h^{b a} k_{b a}\right) d V .
$$


$\S 5$. Closed submanifolds with mean curvature vector parallel with respect to the connection induced in the normal bundle.

We consider a closed orientable submanifold $M^{n}$ of codimension 2 of an $(n+2)$ dimensional Riemannian manifold $M^{n+2}$ and assume that $M^{n}$ admits a vector field $X^{h}$ whose covariant differential along $M^{n}$ is always proportional to the displacement:

$$
\nabla_{c} X^{h}=f B_{c}{ }^{h}
$$

and that the mean curvature vector $\left.(1 / n) g^{c b} \nabla_{c} B_{b}{ }^{h} \neq 0\right)$ is parallel with respect to the connection induced in the normal bundle:

$$
h_{a}{ }^{a}=\text { const. } \neq 0, \quad l_{c}=0 .
$$

Then we have first of all

$$
\int_{M^{n}}\left(n f+\alpha h_{a}^{a}\right) d V=0
$$

We next have from (4.5)

$$
\int_{M^{n}} K_{k j i n} B_{d}^{k} z^{d} B^{j i} C^{h} d V
$$

$$
=\int_{M^{n}}\left(f h_{a}^{a}+\alpha h^{b a} h_{b a}+\beta h^{b a} k_{b a}\right) d V .
$$

Now, forming (5.4)-(5.3) $\times(1 / n) h_{a}{ }^{a}$, we find

$$
\begin{aligned}
& \int_{M^{n}} K_{k j i h} B_{d}{ }^{k} z^{d} B^{j i} C^{h} d V \\
= & \int_{M^{n}}\left[\alpha\left(h^{b a} h_{b a}-\frac{1}{n} h_{b}^{b} h_{a}^{a}\right)+\beta h^{b a} k_{b a}\right] d V,
\end{aligned}
$$

or

$$
\begin{aligned}
& \int_{M^{n}} K_{k j i n} B_{d}{ }^{k} z^{d} B^{j i} C^{h} d V \\
= & \int_{M^{n}}\left[\alpha\left\{\left(h^{b a}-\frac{1}{n} h_{e}^{e} g^{b a}\right)\left(h_{b a}-\frac{1}{n} h_{d}^{d} g_{b a}\right)+k^{b a} k_{b a}\right\}+\left(h^{b a} k_{b a} \beta-k^{b a} k_{b a} \alpha\right)\right] d V .
\end{aligned}
$$

We denote by $X^{\prime h}$ and $X^{\prime h}$ the tangential part and normal part of $X^{h}$ respectively.

Suppose that 


$$
\begin{gathered}
\int_{M^{n}} K_{k j i h} X^{\prime k} B^{j i} C^{h} d V \leqq 0, \\
\alpha>0, \\
h^{b a} k_{b a} \beta-k^{b a} k_{b a} \alpha \geqq 0,
\end{gathered}
$$

that is, the vector

$$
Y^{h}=h^{b a} k_{b a} C^{h}+k^{b a} k_{b a} D^{h}
$$

vanishes or this vector and

$$
X^{\prime \prime h}=\alpha C^{h}+\beta D^{h}
$$

have positive orientation in the normal bundle, or

$$
\begin{gathered}
\int_{M^{n}} K_{k j i h} X^{\prime k} B^{j i} C^{h} d V \geqq 0, \\
\alpha<0, \\
h^{b a} k_{b a} \beta-k^{b a} k_{b a} \alpha \leqq 0,
\end{gathered}
$$

that is, the vector $Y^{h}$ vanishes or $Y^{h}$ and $X^{\prime \prime h}$ have negative orientation in the normal bundle, then we have

$$
h_{c b}-\frac{1}{n} h_{d}^{d} g_{c b}=0, \quad k_{c b}=0,
$$

that is, the submanifold under consideration is totally umbilical. Thus we have

THEOREM 5.1. Let $M^{n}$ be a closed orientable submanifold of codimension 2 of an $(n+2)$-dimensional Riemannian manifold $M^{n+2}$ and assume that $M^{n}$ admits a vector field $X^{h}$ whose covariant differential along $M^{n}$ is always proportional to the displacement. If

(i) the mean curvature vector field $(\neq 0)$ is parallel with respect to the connection induced in the normal bundle,

(ii) $\int_{M^{n}} K_{k j i n} X^{\prime k} B^{j i} C^{h} d V \leqq 0 \quad(\geqq 0)$,

(iii) $\quad \alpha>0 \quad(<0)$,

(iv) $Y^{h}=0$ or $Y^{h}$ and $X^{\prime \prime h}$ have positive (negative) orientation in the normal bundle,

then the submanifold is totally umbilical.

If the submanifold is invariant under the curvature transformation, then we have

$$
K_{k j i h} X^{\prime k} B^{j i} C^{h}=0
$$

and consequently the second condition of Theorem 5.1 is automatically satisfied.

If the ambient Riemannian manifold $M^{n+2}$ admits a scalar function $v$ such that (5. 6)

$$
\nabla_{j} \nabla_{i} v=f(v) g_{j i},
$$


then we have

$$
\nabla_{c} v^{h}=f(v) B_{c}{ }^{h}
$$

along any submanifold, where we have put

$$
v^{h}=v_{i} g^{i n}, \quad v_{i}=\nabla_{i} v .
$$

This equation shows that the vector field $v^{h}$ defined along $M^{n}$ has covariant differential always proportional to the displacement along $M^{n}$.

Thus, under 4 conditions of Theorem 5.1, we have

$$
h_{c b}=\lambda g_{c b}, \quad k_{c b}=0, \quad l_{c}=0,
$$

$\lambda$ being a constant different from zero, and consequently, (3.7) and (3.8),

$$
\nabla_{b} z_{a}=(f+\alpha \lambda) g_{b a}
$$

and

$$
\partial_{b} \alpha+\lambda z_{b}=0
$$

But

$$
z_{b}=B_{b}{ }^{2} v_{i}=\partial_{b} v
$$

and consequently, we have from (3.7) and (5.10),

$$
\alpha+\lambda v=c \text { (constant). }
$$

Thus, from (5.9),

$$
\nabla_{b} \nabla_{a} v=\left(f+c \lambda-\lambda^{2} v\right) g_{b a} .
$$

We examine two cases,

(I) $f=k v, k=$ const. $\neq 0, v \neq$ const. along $M^{n}$.

In this case, we have

$$
\nabla_{b} \nabla_{a} v=\left[-\left(\lambda^{2}-k\right) v+\lambda c\right] g_{b a} .
$$

Here, $\lambda^{2}-k \neq 0$, because if $\lambda^{2}-k=0$, then we have $\nabla_{b} \nabla_{a} v=\lambda c g_{b a}$, from which $g^{b a} \nabla_{b} \nabla_{a} v=n \lambda c$, which, the submanifold being closed, is impossible unless $v=$ constant on $M^{n}$.

Thus, $\lambda^{2}-k$ being different from zero, we have from (5.11)

$$
\nabla_{b} \nabla_{a}\left(v-\frac{\lambda c}{\lambda^{2}-k}\right)=-\left(\lambda^{2}-k\right)\left(v-\frac{\lambda c}{\lambda^{2}-k}\right) g_{b a},
$$

from which

$$
g^{b a} \nabla_{b} \nabla_{a}\left(v-\frac{\lambda c}{\lambda^{2}-k}\right)=-n\left(\lambda^{2}-k\right)\left(v-\frac{\lambda c}{\lambda^{2}-k}\right)
$$


which shows that $\lambda^{2}-k>0$. Thus, by a famous theorem of Obata [5], the submanifold is isometric to a sphere.

(II) $f=k, k=$ constant, $v \neq$ const. along $M^{n}$.

In this case, we have

$$
\nabla_{b} \nabla_{a} v=\left(-\lambda^{2} v+k+c \lambda\right) g_{b a} .
$$

Here $\lambda \neq 0$, because if $\lambda=0$, then we have $v=$ const. along $M^{n}$. Thus we have

$$
\nabla_{b} \nabla_{a}\left(v-\frac{k+c \lambda}{\lambda^{2}}\right)=-\lambda^{2}\left(v-\frac{k+c \lambda}{\lambda^{2}}\right) g_{b a},
$$

from which we conclude that the submanifold is isometric to a sphere. Thus we have

Theorem 5.2. Let $M^{n}$ be a closed orientable submanifold of codimension 2 of an (n+2)-dimensional orientable Riemannian manifold $M^{n+2}$ which admits a scalar function $v$ such that $\nabla_{j} \nabla_{i} v=f(v) g_{j i}$, where $f(v)=k v$, or $k, k$ being $a$ constant, and $v \neq$ const. along $M^{n}$. Then under 4 conditions of Theorem 5.1 where $X^{h}$ $=\left(\nabla_{i} v\right) g^{i n}$, the submanifold is totally umbilical and is isometric to a sphere.

\section{BiBLIOGRAPHY}

[1] Katsurada, Y., Closed submanifolds with constant $\nu$-th mean curvature related with a vector field in a Riemannian manifold. J. of Fac. Sc1., Hokkaido University, Ser. I, 21 (1969), 171-181.

[2] Katsurada, Y., AND H. KôJYô, Some integral formulas for closed submanifolds in a Riemann space. J. of Fac. Sc1., Hokkaido University, Ser. I, 20 (1968), 90-100.

[ 3 ] Katsurada, Y., and T. Nagai, On some properties of a submanifold with constant mean curvature in a Riemann space. J. of Fac. Sci., Hokkaido University, Ser. I, 20 (1968), 79-89.

[4] NAGAI, T., On certain conditions for a submanifold in a Riemannian space to be isometric to a sphere. J. of Fac. Sci., Hokkaido University, Ser. I, 21 (1969), 135-159.

[5] Овата, M., Certain conditions for a Riemannian manifold to be isometric with a sphere. J. Math. Soc. Japan 14 (1962), 333-340.

[6] OKumura, M., Compact orientable submanifold of codimension 2 in an odd dimensional sphere. Tôhoku Math. J. 20 (1968), 8-20.

[ 7 ] OKumura, M., Submanifolds of codimension 2 with certain properties. To appear.

[8] YANO, K., Notes on hypersurfaces in a Riemannian manifold. Canadian J. of Math. 19 (1967), 439-446.

[9] YANO, K., Integral formulas for submanifolds and their applications. To appear in Canadian J. of Math.

Tokyo Institute of Technology, AND

SAITAMa University. 